\title{
Complex Lung Motion Estimation via Adaptive Bilateral Filtering of the Deformation Field
}

\author{
Bartlomiej W. Papież ${ }^{1}$, Mattias Paul Heinrich ${ }^{1}$, \\ Laurent Risser ${ }^{2}$, and Julia A. Schnabel ${ }^{1}$ \\ 1 Institute of Biomedical Engineering, \\ Department of Engineering Science, University of Oxford, UK \\ ${ }^{2}$ CNRS, Institut de Mathématiques de Toulouse (UMR5219), France
}

\begin{abstract}
Estimation of physiologically plausible deformations is critical for several medical applications. For example, lung cancer diagnosis and treatment requires accurate image registration which preserves sliding motion in the pleural cavity, and the rigidity of chest bones. This paper addresses these challenges by introducing a novel approach for regularisation of non-linear transformations derived from a bilateral filter. For this purpose, the classic Gaussian kernel is replaced by a new kernel that smoothes the estimated deformation field with respect to the spatial position, intensity and deformation dissimilarity. The proposed regularisation is a spatially adaptive filter that is able to preserve discontinuity between the lungs and the pleura and reduces any rigid structures deformations in volumes. Moreover, the presented framework is fully automatic and no prior knowledge of the underlying anatomy is required. The performance of our novel regularisation technique is demonstrated on phantom data for a proof of concept as well as 3D inhale and exhale pairs of clinical CT lung volumes. The results of the quantitative evaluation exhibit a significant improvement when compared to the corresponding state-of-the-art method using classic Gaussian smoothing.
\end{abstract}

Keywords: nonrigid registration, respiratory motion, sliding motion modeling, adaptive bilateral filtering.

\section{Introduction}

Image registration is a key processing step in medical image analysis. However, common deformation models such as diffusion, elasticity or fluid methods usually do not reflect the underlying mechanisms (true tissue properties) of the tissue changes between the consecutive volumes. Therefore, additional constraints need to be introduced such as displacement field discontinuities (sliding motion) 8761, rigidity [11] or incompressibility [5]. Applications such as diagnosis and image guided radiotherapy (IGRT) have attracted active research on accurate lung motion estimation over the last years [391.

The sliding motion pattern that naturally occurs at e.g. lungs and liver boundaries has been addressed by various image registration approaches. Direction dependent regularisation [9] decouples diffusion regularisation into normal and 
tangential direction around lung boundaries, while the registration of the remaining part of the volume is based on a classic diffusion model. Similarly, anisotropic diffusion regularisation was utilised for a lung phantom data study in [6]. A different concept was proposed in [8] where the deformation is decomposed into basic components that are then regularised separately. Recently, Large Deformation Diffeomorphic Metric Mapping (LDDMM) was extended towards the piecewisediffeomorphic registration that enables explicit sliding motion modelling 7 . These approaches have some limitations, as they require preprocessing steps in form of segmentation of some structures where sliding motion may occur [6]9], very accurate domain splitting strategy to ensure diffeomorphism [7], or a sliding motion detection system $[9$. For approaches where no segmentations are required or available, the estimated deformations remain smooth at lung boundaries, or discontinuity preserving regularisation is applied to the whole volume domain [8].

Several methodologies that enforce the rigidity on some volume objects have been described in literature. The most related to the method presented in this paper was proposed in [11. It is based on an iterative procedure of adaptive filtering of the deformation field that is employed for the area of rigid objects. The simplicity is however deceptive as it requires a stiffness coefficient which has to be derived either from segmentation or from Hounsfields unit if CT data are available. One such example is the recent work [1, where a fluid registration framework with preservation of topology and rigidity is proposed, which however also relies on the segmentation of the lung surface and bony structures.

This work aims to develop automated method for deformable registration to address both, the problem of sliding motion estimation and the local rigidity preservation. The contributions of this paper are as follows. First, we adapt the bilateral filter technique previously introduced for image filtering and occlusion detection in computer vision applications 1315 to regularise the estimated deformation field in a Demons formulation [12. The classic Gaussian kernel is then replaced by a new kernel that is dependent on the anisotropic diffusion, the intensity and deformation dissimilarity. The presented framework does not require any prior knowledge about the organs' properties and therefore it forms a fully automated technique.

\section{Classic Diffusion Based Regularisation}

In a classic non-linear image registration formulation 416, the optimal displacement field $\hat{\boldsymbol{u}}$ describing the geometrical transformation between a reference image $I_{R}$ and a source image $I_{S}$ is estimated by minimising a global energy:

$$
\hat{\boldsymbol{u}}=\arg \min _{\boldsymbol{u}}\left(\operatorname{Sim}\left(I_{R}, I_{S}(\boldsymbol{u})\right)+\alpha \operatorname{Reg}(\boldsymbol{u})\right)
$$

where Sim is a similarity measure, Reg is a regularisation term, and $\alpha$ is a positive weighting factor. A common choice of similarity criterion is the sum of the squared differences (SSD) and a diffusion regularisation yielding:

$$
\int_{\Omega}\left(I_{R}(\boldsymbol{x})-I_{S}(\boldsymbol{x}+\boldsymbol{u}(\boldsymbol{x}))\right)^{2}+\alpha \operatorname{tr}\left(\nabla \boldsymbol{u}(\boldsymbol{x})^{T} \nabla \boldsymbol{u}(\boldsymbol{x})\right)^{2} d \boldsymbol{x}
$$


The diffusion regularisation is performed as Gaussian smoothing of the deformation field, therefore solving the Euler-Lagrange equations of Eq. (1) can be divided into two steps [15]: finding an update $\boldsymbol{d} \boldsymbol{u}$ that is related to the similarity measure Sim, and performing smoothing of the estimated deformation field using an isotropic Gaussian kernel $G_{i s o}$ instead of explicit regularisation $\operatorname{Reg}(\boldsymbol{u})$ :

$$
\boldsymbol{u}_{\text {new }}(\boldsymbol{x})=G_{\text {iso }} *\left(\boldsymbol{u}_{\text {old }}(\boldsymbol{x}) \circ \boldsymbol{d u}(\boldsymbol{x})\right)
$$

where $\boldsymbol{u}_{n e w}$ is a new estimate of the deformation field, $\boldsymbol{u}_{\text {old }}$ is a deformation field calculated in the previous iteration, and $\circ$ is a composition operation. Filtering the deformation field using an isotropic Gaussian kernel leads to smooth deformations. In medical image registration, this framework can be related to the commonly used Demons algorithm [1214.

\section{Adaptive Bilateral Smoothing}

In order to prevent the deformation field to be smoothed across object boundaries which would not be physically realistic, we propose to replace the standard Gaussian filtering of the deformation field by a more powerful non-linear filtering technique originally proposed for image denoising [13. The bilateral filter smoothes an input image $I_{i}$ by two Gaussian kernels in the following way:

$$
I_{o}(\boldsymbol{x})=\frac{1}{W} \sum_{\boldsymbol{y} \in \mathcal{N}} \underbrace{\exp \left(-\frac{(\boldsymbol{x}-\boldsymbol{y})^{T}(\boldsymbol{x}-\boldsymbol{y})}{2 \sigma_{\boldsymbol{x}}^{2}}\right)}_{G_{\text {iso }}(\boldsymbol{x}, \boldsymbol{y})} \cdot \underbrace{\exp \left(-\frac{\left\|I_{i}(\boldsymbol{x})-I_{i}(\boldsymbol{y})\right\|^{2}}{2 \sigma_{\boldsymbol{r}}^{2}}\right)}_{G_{r}(I(\boldsymbol{x}), I(\boldsymbol{y}))} \cdot I_{i}(\boldsymbol{y})
$$

where $G_{i s o}$ is Gaussian kernel on the spatial domain (with variance $\sigma_{\boldsymbol{x}}^{2}$ ) and $G_{r}$ is another Gaussian kernel but defined on the intensity domain $I_{i}$ (with variance $\left.\sigma_{\boldsymbol{r}}^{2}\right), \boldsymbol{y}$ is a spatial position within the image neighbourhood $\mathcal{N}$, and $W$ is a normalisation factor for this image neighbourhood $\mathcal{N}$. Even though the additional kernel $G_{r}$ does not allow for smoothing when the neighbourhood intensity values are different, direct substitution of the Gaussian kernel by the bilateral kernels in our registration framework can lead to several deformation field discontinuities (motion over-segmentation) in the whole volume domain. This discontinuity can occur at each intensity change and is typical for image-driven regularisation [16]. In addition to this, some organs have very similar intensity values, however they can slide along each other. Therefore, a supplementary kernel is employed [15] and the bilateral filtering of deformation field is extended in the following way:

$$
\begin{array}{r}
\boldsymbol{u}_{n e w}(\boldsymbol{x})=\frac{1}{W} \sum_{\boldsymbol{y} \in \mathcal{N}} G_{i s o}(\boldsymbol{x}, \boldsymbol{y}) \cdot G_{r}(I(\boldsymbol{x}), I(\boldsymbol{y})) \\
\underbrace{\exp \left(-\frac{\left(\boldsymbol{u}_{c u r}(\boldsymbol{x})-\boldsymbol{u}_{c u r}(\boldsymbol{y})\right)^{T}\left(\boldsymbol{u}_{c u r}(\boldsymbol{x})-\boldsymbol{u}_{c u r}(\boldsymbol{y})\right)}{2 \sigma_{\boldsymbol{u}}^{2}}\right)}_{G_{\boldsymbol{u}}(\boldsymbol{u}(\boldsymbol{x}), \boldsymbol{u}(\boldsymbol{y}))} \cdot \boldsymbol{u}_{c u r}(\boldsymbol{y})
\end{array}
$$




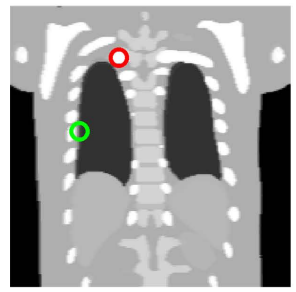

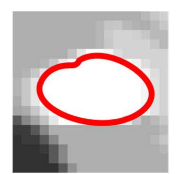

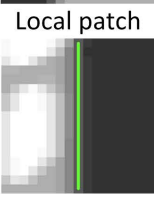

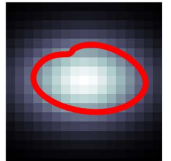

Gani

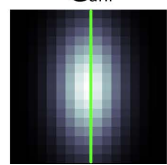

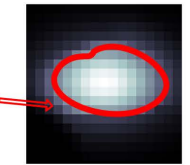

$\mathbf{G}_{\text {ani }} \cdot \mathbf{G}_{\mathrm{r}}$

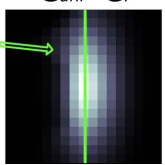

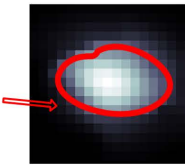

$\mathbf{G}_{\text {ani }} \cdot \mathbf{G}_{r} \cdot \mathbf{G}_{u}$

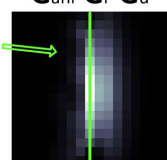

Fig. 1. Comparison between different kernels used for deformation filtering. Two local patches from distinctive areas of the chest were taken from the NCAT data. The patch presenting the chest bone (red circle) and its corresponding kernels are shown in the top row, while the patch including the lung boundary (green circle) and its corresponding kernels are shown in the bottom row.

where $G_{u}$ describes a Gaussian kernel based on the local deformation field dissimilarity and $\boldsymbol{u}_{\text {cur }}(\boldsymbol{x})=\boldsymbol{u}_{\text {old }}(\boldsymbol{x}) \circ \boldsymbol{d u}(\boldsymbol{x})$. Thus, the combination of three kernels $G_{i s o}$, $G_{r}$, and $G_{u}$ leads to a joint image- and deformation-driven regularisation method, which compromises mutual benefits such as discontinuous deformation without motion over-segmentation. Finally, we can also replace the isotropic Gaussian kernel $G_{i s o}$ by an oriented Gaussian kernel $G_{a n i}$. After that substitution, the kernel $G_{a n i}$ varies at different image position $\boldsymbol{x}$ with respect to the diffusion tensor $\boldsymbol{D}$. The diffusion tensor $\boldsymbol{D}$ for $n$-dimensional volumes is defined as [4]:

$$
\boldsymbol{D}=\frac{\left(\lambda+\|\nabla I\|^{2}\right) \boldsymbol{I} \boldsymbol{d}-\nabla I \nabla I^{T}}{(n-1)\|\nabla I\|^{2}+n \lambda}
$$

where $\nabla I$ is the gradient of image $I, \lambda$ is an (an)isotropy parameter, $\boldsymbol{I} \boldsymbol{d}$ is a $n \times n$ identity matrix. As can be expected, if the intensity values around point $\boldsymbol{x}$ are (close to) constant $(\|\nabla I\| \approx 0)$, the eigenvalues of $\boldsymbol{D}$ are equal and the kernel $G_{a n i}$ is equivalent to the isotropic kernel $G_{i s o}$.

Examples of different kernels are presented in Fig. 11. Filtering the deformation field with the anisotropic Gaussian kernel leads to smooth flow at lung boundaries (Fig. 1 bottom row) as the kernel averages the deformation field between neighbourhood areas. Whereas the bilateral filter working on both the spatial $G_{a n i}$ and intensity domain $G_{r}$ improves the shape of the kernel, still some deformation field averaging is done (see area marked by arrows). While better separation between different structures can be only captured by limiting the size of the bilateral filter using only an intensity kernel (lower value of variance $\sigma_{r}^{2}$ ), it will lead to the aforementioned motion over-segmentation problem. Such example of motion over-segmentation in case of lung data registration is illustrated in Fig. 2r. The proposed composition of three kernels $G_{a n i}, G_{r}$ and $G_{u}$ produces a kernel which visually has better overlap with the underlying anatomical structure than the two others. Similarly, the kernel comprising all of $G_{a n i}, G_{r}$ and $G_{u}$ acts mostly inside the rigid structure (Fig. 1 top row). 
Table 1. Average ratio of overlap obtained for the NCAT data set using the Demon framework with four different smoothing kernels. The last column shows statistical significance of improvement between iso-dem compared to others methods.

\begin{tabular}{cllll}
\hline & \multicolumn{1}{c}{ lungs } & \multicolumn{1}{c}{ liver } & \multicolumn{1}{c}{ ribs } & \multicolumn{1}{c}{ p-value } \\
\hline iso-dem & $0.86 \pm 0.08$ & $0.89 \pm 0.10$ & $0.76 \pm 0.05$ & $\mathrm{~N} / \mathrm{A}$ \\
ani-dem & $0.90 \pm 0.05$ & $0.91 \pm 0.07$ & $0.79 \pm 0.05$ & 0.475 \\
iso-bil & $\mathbf{0 . 9 2} \pm \mathbf{0 . 0 2}$ & $\mathbf{0 . 9 3} \pm \mathbf{0 . 0 5}$ & $\mathbf{0 . 8 0} \pm \mathbf{0 . 0 5}$ & 0.061 \\
ani-bil & $\mathbf{0 . 9 2} \pm \mathbf{0 . 0 2}$ & $\mathbf{0 . 9 3} \pm \mathbf{0 . 0 5}$ & $\mathbf{0 . 8 0} \pm \mathbf{0 . 0 5}$ & 0.067 \\
\hline
\end{tabular}

\section{Results}

Materials. The proposed approach is assessed using two publicly available data sets. The first data set is a set of synthetically generated 4D CT volumes modelling consecutive respiratory cycle phases from the NCAT phantom [10]. The spatial resolution of the data is $2.0 \times 2.0 \times 2.0 \mathrm{~mm}^{3}$. The second data set consists of ten 4D CT data from the Dir-Lab data set 2. The spatial resolution of that data varies between $0.97 \times 0.97 \times 2.5 \mathrm{~mm}^{3}$ and $1.16 \times 1.16 \times 2.5 \mathrm{~mm}^{3}$. For all data sets, the end-of-inspiration volume was chosen as a reference, and the end-of-expiration volume as a source image.

Experiments. For quantitative evaluation of the proposed regularisation filters, a Demon approach with an update composition scheme was implemented (see 14] for details). Four different kernels for smoothing the deformation field were used for comparison: isotropic Gaussian $G_{i s o}$ (iso-dem), anisotropic Gaussian $G_{a n i}(\mathbf{a n i}-\mathbf{d e m})$, bilateral kernel with isotropic Gaussian $G_{i s o} \cdot G_{r} \cdot G_{\boldsymbol{u}}$ (iso-bil) and with anisotropic Gaussian $G_{a n i} \cdot G_{r} \cdot G_{\boldsymbol{u}}$ (ani-bil). In most cases, the registration with a bilateral filter without deformation similarity kernel $G_{i s o} \cdot G_{r}$ produces unrealistic deformation fields (see example in Fig. 2re), therefore the quantitative results obtained are not included. Additionally, a two-sample Wilcoxon rank sum test was performed between iso-dem and the other evaluated methods. Filtering of the deformation field was performed once after each update of deformation field, but in principle it could be done several times at each iteration following the approach presented in [11. Although the best design parameters $\left(\sigma_{\boldsymbol{x}}, \sigma_{r}, \sigma_{\boldsymbol{u}}\right.$, $\lambda$ ) were determined empirically by an extensive search over parameter space for each method, experiments on both data sets showed that the proposed algorithm is very robust to their choice, hence these parameters do not need to be tuned for each volume separately (for this particular application i.e. lung CT).

Results on NCAT Data. The ratio of overlap (RO) for the organ of interest (lungs, liver, ribs) was calculated and the registration outcomes for the NCAT data are presented in Tab. 1. The RO exhibits an improvement for methods based on the bilateral filtering when compared with the Gaussian smoothing. Although this does not necessarily ensure deformation field plausibility, it can highlight differences between methods in terms of the anatomical correspondence. 
Results on CT Dir-Lab Data. The TRE was calculated for the landmarks which are included in this data set (300 per case) and the results of quantitative evaluation can be found in Tab. 2. The initial average TRE is $8.46 \pm 5.48 \mathrm{~mm}$. As can be seen, the deformation fields obtained using both frameworks based on the bilateral filtering produce significantly lower TRE when compared to the classic Demon algorithm. Contrary to expectation, the bilateral filtering with anisotropic kernel $G_{a n i}$ performs slightly worse than the method with isotropic kernel $G_{\text {iso }}$. This indicates that the bilateral filtering with isotropic Gaussian kernel can effectively adapt smoothing across different structures. Moreover, the proposed methods based on bilateral filtering yields a lower TRE $(2.34 \mathrm{~mm})$ whereas the classic Demon has a $\mathrm{TRE}=2.88 \mathrm{~mm}$. The results reported in the literature 9] were $3.02 \mathrm{~mm}$ for diffusion regularisation and $2.13 \mathrm{~mm}$ for direction dependent regularisation. It must be noted that further improvement might be expected when a more advanced similarity Sim would be applied to capture local intensity variations apparent in the Dir-Lab data due to lung compression [2].

Fig. 2] is an illustrative example of the deformation field magnitudes when registering case $c 5$ from the Dir-Lab data set using different smoothing kernels. The results from the quantitative evaluation (shown in fifth row of Tab. 22) exhibit a statistically significant improvement in terms of the TRE between different methods, and consequently some noticeable differences between the estimated deformation fields can be identified especially close to the lung boundaries (compare Fig. 2b and Fig. 2 $\mathrm{b}$, and its corresponding zoomed images in Fig. 2f and Fig. 2h). Employing bilateral filtering derived both from intensity and deformation field similarity preserves discontinuity between the lungs and the pleura, while satisfying smoothness of the deformation field inside the lungs. Contrary, applying the bilateral filter based only on intensity difference generates discontinuities inside and outside the pleural cavity (depicted by black arrows in Fig.22).

Table 2. Target Registration Error and its standard deviations obtained for Dir-Lab data set using the Demon framework with four different smoothing kernels. The last column shows statistical significance of improvement between iso-dem compared to others methods with $p$-value level below 0.05 (marked as $(+)$ ). The proposed iso-bil achieves the lowest average TRE among all methods.

\begin{tabular}{ccccccc}
\hline No. data & before & iso-dem & ani-dem & iso-bil & ani-bil & sign. \\
\hline c1 & $3.89 \pm 2.78$ & $1.08 \pm 0.57$ & $1.09 \pm 0.58$ & $1.05 \pm 0.54$ & $1.07 \pm 0.57$ & --- \\
c2 & $4.34 \pm 3.90$ & $1.11 \pm 0.64$ & $1.10 \pm 0.63$ & $1.08 \pm 0.58$ & $1.09 \pm 0.60$ & --- \\
c3 & $6.94 \pm 4.05$ & $1.54 \pm 0.98$ & $1.52 \pm 0.91$ & $1.47 \pm 0.86$ & $1.49 \pm 0.89$ & -++ \\
c4 & $9.83 \pm 4.86$ & $2.38 \pm 2.04$ & $2.38 \pm 2.03$ & $2.28 \pm 1.82$ & $2.40 \pm 2.04$ & -+- \\
c5 & $7.48 \pm 5.51$ & $2.26 \pm 1.93$ & $2.22 \pm 1.90$ & $2.04 \pm 1.71$ & $2.13 \pm 1.78$ & -++ \\
c6 & $10.9 \pm 6.97$ & $3.26 \pm 2.84$ & $3.14 \pm 2.67$ & $2.72 \pm 2.01$ & $3.05 \pm 2.49$ & +++ \\
c7 & $11.0 \pm 7.43$ & $3.81 \pm 3.69$ & $3.62 \pm 3.47$ & $3.14 \pm 2.76$ & $3.53 \pm 3.33$ & +++ \\
c8 & $15.0 \pm 9.01$ & $8.22 \pm 8.27$ & $7.73 \pm 8.26$ & $4.99 \pm 5.14$ & $7.19 \pm 7.67$ & +++ \\
c9 & $7.92 \pm 3.98$ & $2.56 \pm 2.02$ & $2.46 \pm 1.89$ & $2.08 \pm 1.45$ & $2.36 \pm 1.79$ & +++ \\
c10 & $7.30 \pm 6.35$ & $2.65 \pm 3.27$ & $2.61 \pm 3.21$ & $2.45 \pm 2.78$ & $2.57 \pm 3.11$ & -++ \\
\hline mean & $8.46 \pm 5.48$ & $2.88 \pm 2.07$ & $2.79 \pm 1.92$ & $\mathbf{2 . 3 4} \pm \mathbf{1 . 1 6}$ & $2.67 \pm 1.77$ &
\end{tabular}



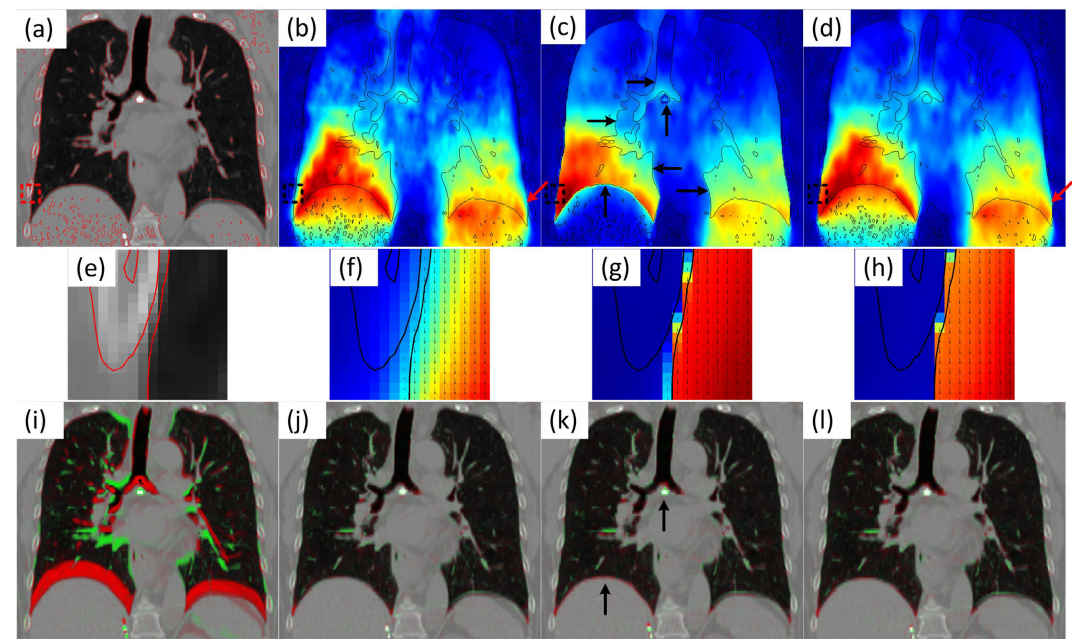

Fig. 2. Results for case $c 5$ of the Dir-Lab data set. (a) coronal view of the reference image with the corresponding contours. The magnitude of the deformation fields estimated using: (b) isotropic Gaussian kernel $G_{i s o}$ (Demon), (c) original bilateral kernel $G_{\text {iso }} \cdot G_{r}$, (d) the proposed bilateral kernel $G_{i s o} \cdot G_{r} \cdot G_{\boldsymbol{u}}$, (e)-(h) zoomed images of the region of interest (labelled by squared box in the top row), the intensity differences between input images (i) before registration, and after using (j) iso-dem, (k) original bilateral kernel $G_{i s o} \cdot G_{r}$, and (l) iso-bil. Registration using iso-bil yields smooth deformation inside the pleura cavity whilst preserving sliding motion at the lung boundary.

\section{Discussion and Conclusions}

This paper presents an image registration framework which is able to estimate deformation fields preserving both the sliding motion in the cavity of the pleura whilst preserving the rigidity of the chest bones and yielding desirable smooth deformation field inside the lungs. The overall deformation field is regularised within a one step procedure that is performed via adaptive deformation field filtering. The kernel which is used for the purpose of deformation field filtering, is based on three components: spatial smoothness, local image intensity and deformation field similarity. Evaluation of the proposed regularisation scheme was done both on the NCAT phantom data and clinical lung CT data. In cases where noticeable sliding motion occurs in the data, the presented results exhibit significant improvements when the new filtering procedure is applied compared to the classic Gaussian smoothing. Moreover, for non-sliding cases (where statistical significance of the improvements for the proposed filtering procedure was not achieved) the slightly lower average TRE was obtained, and in addition, the visual inspection of the estimated deformation fields still exhibited physiologically more plausible results. Future work will perform a sensitivity analysis of design parameters of the proposed filtering procedure such as $\sigma_{\boldsymbol{x}}, \sigma_{r}, \sigma_{\boldsymbol{u}}$ to this 
application, and comparison to methods that require segmentation or explicit sliding motion detection to achieve desired properties [79].

Acknowledgements. We would like to acknowledge funding from the CRUK/ EPSRC Oxford Cancer Imaging Centre. JAS and LR also wish to acknowledge the INSMI-CNRS/John Fell Oxford University Press (OUP) Research Fund.

\section{References}

1. Baluwala, H., Risser, L., Schnabel, J.A., Saddi, K.: Toward physiologically motivated registration of diagnostic CT and PET/CT of lung volumes. Med. Phys. 40, 021903 (2013)

2. Castillo, R., Castillo, E., Guerra, R., Johnson, V., McPhail, T., Garg, A., Guerrero, T.: A framework for evaluation of deformable image registration spatial accuracy using large landmark point sets. Phys. Med. Biol. 54, 1849-1870 (2009)

3. Ehrhardt, J., Werner, R., Schmidt-Richberg, A., Handels, H.: Statistical modeling of $4 \mathrm{D}$ respiratory lung motion using diffeomorphic image registration. IEEE Trans. Med. Imag. 30, 251-265 (2011)

4. Hermosillo, G., Chefd'Hotel, C., Faugeras, O.: Variational Methods for Multimodal Image Matching. Int. J. Comput. Vision 50, 329-343 (2002)

5. Mansi, T., Pennec, X., Sermesant, M., Delingette, H., Ayache, N.: iLogDemons: A Demons-Based Registration Algorithm for Tracking Incompressible Elastic Biological Tissues. Int. J. Comput. Vision 92, 92-111 (2011)

6. Pace, D.F., Enquobahrie, A., Yang, H., Aylward, S.R., Niethammer, M.: Deformable image registration of sliding organs using anisotropic diffusive regularization. In: IEEE ISBI, pp. 407-413 (2011)

7. Risser, L., Vialard, F.X., Baluwala, H., Schnabel, J.A.: Piecewise-diffeomorphic image registration: Application to the motion estimation between 3D CT lung images with sliding conditions. Med. Image Anal. 17, 182-193 (2013)

8. Ruan, D., Esedoglu, S., Fessler, J.A.: Discriminative Sliding Preserving Regularization in Medical Image Registration. In: IEEE ISBI, pp. 430-433 (2009)

9. Schmidt-Richberg, A., Werner, R., Handels, H., Ehrhardt, J.: Estimation of slipping organ motion by registration with direction-dependent regularization. Med. Image Anal. 16, 150-159 (2012)

10. Segars, W.P.: Development and application of the new dynamic NURBS-based cardiac-torso (NCAT) phantom. PhD thesis, University of North Carolina (2001)

11. Staring, M., Klein, S., Pluim, J.P.W.: Nonrigid registration with tissue-dependent filtering of the deformation field. Phys. Med. Biol. 52, 6879-6892 (2007)

12. Thirion, J.P.: Image matching as a diffusion process: an analogy with Maxwell's demons. Med. Image Anal. 2, 243-260 (1998)

13. Tomasi, C., Manduchi, R.: Bilateral filtering for gray and color images. In: IEEE ICCV, pp. 839-846 (1998)

14. Vercauteren, T., Pennec, X., Perchant, A., Ayache, N.: Diffeomorphic Demons: Efficient non-parametric image registration. NeuroImage 45, 61-72 (2009)

15. Xiao, J., Cheng, H., Sawhney, H., Rao, C., Isnardi, M.: Bilateral filtering-based optical flow estimation with occlusion detection. In: Leonardis, A., Bischof, H., Pinz, A. (eds.) ECCV 2006, Part I. LNCS, vol. 3951, pp. 211-224. Springer, Heidelberg (2006)

16. Zimmer, H., Bruhn, A., Weickert, J.: Optic Flow in Harmony. Int. J. Comput. Vision 93, 368-388 (2011) 